\title{
Regularization method for the radially symmetric inverse heat conduction problem
}

\section{Djerrar, L Alem and L Chorfi*}

\section{"Correspondence:}

I_chorfi@hotmail.com

Lab. LMA, Badji Mokhtar University,

Annaba, Algeria

\begin{abstract}
We consider an axisymmetric inverse problem for the heat equation inside the cylinder $a \leq r \leq b$. We wish to determine the surface temperature on the interior surface $\{r=a\}$ from the Cauchy data on the exterior surface $\{r=b\}$. This problem is ill-posed. Using the Laplace transform, we solve the direct problem. Then the inverse problem is reduced to a Volterra integral equation of the first kind. A standard Tikhonov regularization method is applied to the approximation of this integral equation when the data is not exact. Some numerical examples are given to illustrate the stability of the proposed method.
\end{abstract}

MSC: 35K05; 65N06; 35R30; 44A10

Keywords: ill-posed problem; radially symmetric heat equation; Laplace transform; Tikhonov regularization

\section{Introduction}

The inverse heat conduction problems (IHCPs) have many applications in different branches of science and technology. It consists in determining the temperature and heat flux on both sides of the boundary when one side is inaccessible to measurements.

The mathematical model of our problem can be described by the axisymmetric heat equation

$$
\frac{\partial u}{\partial t}(r, t)=\frac{\partial^{2} u}{\partial r^{2}}(r, t)+\frac{1}{r} \frac{\partial u}{\partial r}(r, t), \quad r \in(a, b), t>0,
$$

with the boundary conditions

$$
u(b, t)=g(t), \quad \frac{\partial u}{\partial r}(b, t)=h(t), \quad t \geq 0,
$$

and the initial condition

$$
u(r, 0)=0, \quad r \in(a, b)
$$

where $r$ is the radial coordinate, and $0<a<b$.

Our purpose is to determine the boundary condition

$$
u(a, t)=f(t), \quad t \geq 0
$$

(c) The Author(s) 2017. This article is distributed under the terms of the Creative Commons Attribution 4.0 International License (http://creativecommons.org/licenses/by/4.0/), which permits unrestricted use, distribution, and reproduction in any medium, provided you give appropriate credit to the original author(s) and the source, provide a link to the Creative Commons license, and indicate if changes were made. 
from the measured Cauchy data $(g, h)$. It is known that this problem is severely ill-posed in the sense that if the solution exists, then it does not depend continuously on the data $g$. Indeed, a small perturbation in the data may cause dramatically a large error in the solution $u(\cdot, t)$. Hence, a regularization method is needed.

The standard problem of the heat conduction in Cartesian coordinates $u_{t}=u_{x x}, x \in$ $[0,1], t>0$, with the data $u(0, t)=f(t)$ and $u_{x}(0, t)=0$ is well studied by various methods. The Fourier method was used in [1-3]. The mollification method and projection regularization based on the Laplace and Fourier transforms are applied respectively in [4] and [5]. For axisymmetric problems, we should mention recent articles. In [6, 7], the authors consider an axisymmetric IHCP of determining the surface temperature from a fixed location inside a cylinder. In $[8,9]$, the authors investigated the case of identifying a source from the final data. Xiong [10] studied the problem of identifying a boundary condition by the method of quasi-reversibility. A modified Tikhonov regularization method was applied for an axisymmetric backward heat equation in [11]. Lesnic et al. [12] applied the method of fundamental solutions (MFS) (with a Tikhonov regularization) to the radially symmetric inverse heat conduction problem (IHCP) analogous to our problem. Inverse problems for fractional diffusion equations are studied by many authors; for example, we mention the recent article [13].

In this paper, we formulate problem (1)-(4) as an integral equation of the first-kind of Volterra type. Then we use the Tikhonov regularization method to approximate this equation. To the author's knowledge, there are no papers devoted to IHCP with radial axisymmetry using the Laplace transform. Our contribution can therefore be considered as a generalization of the paper [5] to the axisymmetric case.

This paper is organized as follows. In Section 2, we give a representation of the solution of the direct problem using the Laplace transform. Then finite difference method (FDM) is applied to give numerical approximation. In Section 3, our inverse problem is reduced to the integral equation of Volterra type; then we apply the Tikhonov regularization method to compute the boundary temperature $u(a, t)=f(t)$ from the Cauchy data $u(b, t)=g(t)$, $u_{r}(b, t)=0$ and give some numerical results. Finally, in Section 4 , we present a conclusion.

\section{Direct problem}

Problem (1)-(2) can be reduced to an integral equation. For this, we assume that $u(a, t)=$ $f(t)$ is known and (for simplicity) $h(t)=0$. We consider the following direct problem: given $f$, find $u(x, t)$ such that

$$
\begin{aligned}
& \frac{\partial u}{\partial t}(r, t)=\frac{\partial^{2} u}{\partial r^{2}}(r, t)+\frac{1}{r} \frac{\partial u}{\partial r}(r, t), \quad r \in(a, b), t>0, \\
& u(a, t)=f(t), \quad \frac{\partial u}{\partial r}(b, t)=0, \quad t \geq 0 \\
& u(r, 0)=0, \quad r \in(a, b) .
\end{aligned}
$$

We have the following uniqueness theorem.

Theorem 1 Problem (5) has at most one solution in the space

$$
\mathcal{H}=C^{0}\left(\left[0,+\infty\left[, H^{2}(] a, b[)\right) \cap C^{1}(] 0,+\infty\left[, L^{2}(] a, b[)\right) .\right.\right.
$$


Proof Let $u(r, t)$ be a solution of the homogeneous problem (5) (with $f=0$ ). Multiplying PDE by $r u$ and integrating by parts over the interval $(a, b)$, we obtain the identity

$$
\int_{a}^{b} \frac{\partial u}{\partial t} u r d r+\int_{a}^{b}\left(\frac{\partial u}{\partial r}\right)^{2} r d r=0
$$

We set $E(t)=\int_{a}^{b} u^{2} r d r, t \geq 0$, which leads to

$$
\frac{d E}{d t}=2 \int_{a}^{b} u_{t} u r d r=-2 \int_{a}^{b}\left(u_{r}\right)^{2} r d r \leq 0,
$$

and therefore $E(t)=E(0)=0$ for all $t \geq 0$, and $u(r, t)=0, r \in(a, b), t \geq 0$.

\subsection{Reconstruction of the solution}

We use the Laplace transform (with respect to the variable $t$ ) for the representation of the solution.

Let $f(t), t \geq 0$, be a continuous function of slow growth, which means that there exist two constants $C \geq 0$ and $\sigma \geq 0$ such that $|f(t)| \leq C e^{\sigma t}$ for $t>0$.

The Laplace transform $F(s)=\mathcal{L} f(s)$ of $f(t)$ is defined by

$$
F(s)=\int_{0}^{+\infty} e^{-s t} f(t) d t, \quad \Re(s)>\sigma
$$

which is an analytic function in the half-plane $\Re(s)>\sigma$, and the inverse Laplace transform is given by the complex integral [14]

$$
f(t)=\mathcal{L}^{-1}(F)(t)=\frac{1}{2 \pi i} \int_{\sigma-i \infty}^{\sigma+i \infty} e^{s t} F(s) d s, \quad t>0
$$

Let $U(r, s)=\mathcal{L} u(r, \cdot)$ and $F(s)=\mathcal{L} f(s)$. Problem (5) can be formulated as follows:

$$
\begin{aligned}
& U^{\prime \prime}+\frac{1}{r} U^{\prime}-s U=0, \quad r \in(a, b), \\
& U(a, s)=F(s), \quad U^{\prime}(b, s)=0,
\end{aligned}
$$

where $U^{\prime}=\frac{\partial U}{\partial r}$.

The first equation in (6) is the modified Bessel differential equation with the general solution

$$
U(r, s)=C_{0} I_{0}(r \sqrt{s})+C_{1} K_{0}(r \sqrt{s}) .
$$

Then the solution of problem (6) is given by

$$
U(r, s)=F(s) \frac{W(r, s)}{W(a, s)}
$$

with

$$
W(r, s)=I_{1}(b \sqrt{s}) K_{0}(r \sqrt{s})+K_{1}(b \sqrt{s}) I_{0}(r \sqrt{s}),
$$


where $I_{v}$ and $K_{v}$ are the modified Bessel functions of the first and second kind, respectively [15].

Applying the inverse Laplace transform and the convolution theorem [14], we obtain:

$$
u(r, t)=\int_{0}^{t} f(\tau) k(r, t-\tau) d \tau
$$

with the kernel

$$
k(r, t)=\frac{1}{2 \pi i} \int_{-i \infty}^{+i \infty} \frac{W(r, s)}{W(a, s)} e^{s t} d s, \quad t>0 .
$$

Lemma 1 The heat kernel $k(r, t)$ satisfies the properties

(i)

$$
k(r, t)=\sum_{n=1}^{\infty} \beta_{n} \frac{W\left(r, s_{n}\right)}{W^{\prime}\left(a, s_{n}\right)} e^{s_{n} t} \quad \text { for } r>a, t>0,
$$

with

$$
\begin{aligned}
W\left(r, s_{n}\right)= & J_{0}\left(\frac{r}{a} \beta_{n}\right) Y_{1}\left(\lambda \beta_{n}\right)-J_{1}\left(\lambda \beta_{n}\right) Y_{0}\left(\frac{r}{a} \beta_{n}\right), \quad \lambda=\frac{b}{a}, \\
W^{\prime}\left(a, s_{n}\right)= & J_{1}\left(\beta_{n}\right) Y_{1}\left(\lambda \beta_{n}\right)-J_{1}\left(\lambda \beta_{n}\right) Y_{1}\left(\beta_{n}\right) \\
& +\lambda\left[J_{0}\left(\lambda \beta_{n}\right) Y_{0}\left(\beta_{n}\right)-J_{0}\left(\beta_{n}\right) Y_{0}\left(\lambda \beta_{n}\right)\right],
\end{aligned}
$$

where $J_{v}$ and $Y_{v}$ denote the Bessel functions of the first and second kind, respectively, and $s_{n}=-\beta_{n}^{2}, n=1,2, \ldots$, is the sequence of the zeros of $W(a, s)$.

(ii) for all $n \in \mathbf{N}$ and $a<r \leq b$,

$$
\frac{\partial^{n} k}{\partial t^{n}}(r, 0)=0
$$

Proof (i) Considering the contour $C_{R}=[-i R, i R] \cup\left\{R e^{i \theta}, \theta \in\left[\frac{\pi}{2}, \frac{3 \pi}{2}\right]\right\}$ and using the asymptotic formula $\left|\frac{W(r, s)}{W(a, s)} e^{s t}\right|=O\left(e^{-\mu|s|^{1 / 2}}\right)$ as $|s| \rightarrow \infty$ (see Appendix 1), we can express the previous integral for $t>0$ and $a<r \leq b$ as follows:

$$
k(r, t)=\frac{1}{2 \pi i} \lim _{R \rightarrow \infty} \int_{C_{R}} \frac{W(r, s)}{W(a, s)} e^{s t} d s .
$$

The function $W(a, s)$ is analytic with respect to the variable $p=\sqrt{s}$ and possesses a sequence of simple roots $p_{n}$ located on the imaginary axis such that $p_{n}=-i \beta_{n}$ where $\beta_{n} \simeq\left(n-\frac{1}{2}\right) \frac{a \pi}{b-a}, n=1,2, \ldots$ (see Appendix 2). Formula (11) follows from the Cauchy theorem.

(ii) Deriving the integral (10) with respect to $t$, for all $n \in \mathbf{N}$, we obtain

$$
\frac{\partial^{n} k}{\partial t^{n}}(r, 0)=\frac{1}{2 \pi i} \int_{-i \infty}^{+i \infty} s^{n} \frac{W(r, s)}{W(a, s)} d s, \quad r>a .
$$

The function $G_{n}(r, s)=s^{n} \frac{W(r, s)}{W(a, s)}$ is analytic in the half-plane $\Re(s) \geq 0$ except at the origin, where $\lim _{s \rightarrow 0} G_{0}(r, s)=1$ and $\lim _{s \rightarrow 0} G_{n}(r, s)=0$ for $n \geq 1$, and has the behavior $\left|G_{n}(r, s)\right|=$ 
$O\left(\rho^{n} \exp \left[-(r-a) \rho^{1 / 2} \cos \frac{\theta}{2}\right]\right)$ as $\rho=|s| \rightarrow+\infty$ uniformly for $\theta \in\left[-\frac{\pi}{2}, \frac{\pi}{2}\right]$. Then we can use the Cauchy theorem with an adequate contour to have

$$
\left.\left.\frac{\partial^{n} k}{\partial t^{n}}(r, 0)=0 \quad \text { for } r \in\right] a, b\right]
$$

As a consequence, the integral (9) is written as the formal series

$$
u(r, t)=\sum_{n=1}^{\infty} \beta_{n} \frac{W\left(r, s_{n}\right)}{W^{\prime}\left(a, s_{n}\right)} e^{s_{n} t} \int_{0}^{t} f(\tau) e^{-s_{n} \tau} d \tau, \quad r>a, t>0
$$

Theorem 2 Assume that $f(t) \in C^{1}([0,+\infty[)$ is such that $f(0)=0$ and $f(t)=0$ for $t \geq T$. Then series (17) converges in $L^{2}(] a, b[)$ for all $t \geq 0$ and defines a solution of problem (5) belonging to $\mathcal{H}$.

Proof Integrating by parts in (17), we can write $u$ in the form

$$
u(r, t)=-\sum_{n=1}^{\infty} \frac{1}{\beta_{n}} \frac{W\left(r, s_{n}\right)}{W^{\prime}\left(a, s_{n}\right)} e^{s_{n} t} \int_{0}^{t} f^{\prime}(\tau) e^{-s_{n} \tau} d \tau+f(t) \sum_{n=1}^{\infty} \frac{1}{\beta_{n}} \frac{W\left(r, s_{n}\right)}{W^{\prime}\left(a, s_{n}\right)}
$$

Using the asymptotic behavior (see Appendix 1)

$$
\frac{W\left(r, s_{n}\right)}{W^{\prime}\left(a, s_{n}\right)}=\frac{(-1)^{n} \sqrt{a}}{\sqrt{r}(1-\lambda)} \cos \left(\frac{b-r}{a} n\right)+O\left(\frac{1}{n}\right) \quad(n \rightarrow \infty)
$$

and the estimate

$$
\left|\int_{0}^{t} f(\tau) e^{s_{n}(t-\tau)} d \tau\right| \leq M / \beta_{n}^{2}, \quad M=\sup |f(t)|
$$

we see that the first series in (18) is uniformly convergent and the second term is of the same nature as the series $\sum \frac{\alpha_{n}}{\sqrt{r}} \cos \left(n\left(\lambda-\frac{r}{a}\right)\right), \alpha_{n}=\frac{(-1)^{n}}{\beta_{n}}$. Let us show that last series converges in $L^{2}(] a, b[, r d r)$. Indeed, the partial sum $S_{N}(r)=\sum_{n=0}^{N} \frac{\alpha_{n}}{\sqrt{r}} \cos \left(\frac{(b-r) n}{a}\right)$ is a Cauchy sequence, that is, for all $q \geq 1$,

$$
\begin{aligned}
& \int_{a}^{b}\left|S_{N+q}-S_{N}\right|^{2} r d r \\
& \quad=\int_{a}^{b}\left|\sum_{n=N}^{N+q-1} \alpha_{n} \cos \left(n\left(\lambda-\frac{r}{a}\right)\right)\right|^{2} d r=\int_{0}^{\lambda-1}\left|a \sum_{n=N}^{N+q-1} \alpha_{n} \cos (n z)\right|^{2} d z \\
& \quad \leq l \int_{0}^{\pi}\left|\sum_{n=N}^{N+q-1} \alpha_{n} \cos (n z)\right|^{2} d z=\frac{l \pi}{2} \sum_{n=N}^{N+q-1}\left|\alpha_{n}\right|^{2} \rightarrow 0 \quad(\text { as } N \rightarrow \infty)
\end{aligned}
$$

In the last step, we used the orthogonality of the system $\{\cos (n z)\}$ in $L^{2}(] 0, \pi[)$, and $l$ is an integer chosen such that $\lambda-1 \leq l \pi$. Furthermore,

$$
\frac{\partial u}{\partial t}(r, t)=\sum_{n=1}^{\infty} \beta_{n} \frac{W\left(r, s_{n}\right)}{W^{\prime}\left(a, s_{n}\right)} \int_{0}^{t} f^{\prime}(\tau) e^{s_{n}(t-\tau)} d \tau
$$


which converges as the series $\sum \alpha_{n} \cos \frac{(b-r) n}{a}$ with $\left|\alpha_{n}\right| \leq M^{\prime} / \beta_{n}, M^{\prime}=\sup \left|f^{\prime}(t)\right|$. Therefore $\frac{\partial u}{\partial t} \in L^{2}(] a, b[)$. Now we show that $u$ is a weak solution of the PDE in problem (5). For this, let us consider the sequence

$$
u_{N}(r, t)=\int_{0}^{t} f(\tau) k_{N}(r, t-\tau) d \tau, \quad N \in \mathbf{N}
$$

with

$$
k_{N}(r, t)=\sum_{n=1}^{N} \beta_{n} \frac{W\left(r, s_{n}\right)}{W^{\prime}\left(a, s_{n}\right)} e^{s_{n} t} .
$$

Defining the differential operator $P v:=\frac{\partial v}{\partial t}-\frac{\partial^{2} v}{\partial r^{2}}-\frac{1}{r} \frac{\partial v}{\partial r}$, we have

$$
P u_{N}(r, t)=f(t) k_{N}(r, 0)+\int_{0}^{t} f(\tau) P k_{N}(r, t-\tau) d \tau
$$

However, $P k_{N}(r, t)=0$, and then $P u_{N}(r, t)=f(t) k_{N}(r, 0)$. From Lemma 1 it follows that

$$
\lim _{N \rightarrow \infty} k_{N}(r, 0)=k(r, 0)=0 .
$$

On the other hand, $\left.P: \mathcal{D}^{\prime}(\mathbf{Q}) \rightarrow \mathcal{D}^{\prime}(\mathbf{Q}), \mathbf{Q}=\right] a, b[\times] 0,+\infty[$, is a continuous operator. Then $P u=\lim _{N \rightarrow \infty} P u_{N}=0$. We now show that $u \in \mathcal{H}$. Since $\frac{\partial}{\partial r}\left(r u_{r}\right)=r u_{r r}+u_{r}=r u_{t}$ in $\mathcal{D}^{\prime}(] a, b[)$ at fixed $t$, we have $u_{r} \in H^{1}(] a, b[)$ and $u(\cdot, t) \in H^{2}(] a, b[)$. Finally, it is easy to verify that $t \mapsto u(\cdot, t)$ is $C^{1}$ from $] 0, T\left[\right.$ to $L^{2}(] a, b[)$.

\section{Remark 1}

- If $f \in L^{2}\left(\mathrm{R}_{+}\right)$(not smooth), then the differentiation of series (17) with respect to the variable $r$ or $t$ presents some difficulties. We can only say from the previous proof that $u$ is a weak solution in $L^{2}(] a, b[)$. However, if we know that $u$ is differentiable with respect to $t$, then $u$ is regular in both variables $(r, t)$.

- For the numerical computation, the integral $g_{n}(t)=\int_{0}^{t} f(\tau) e^{s_{n}(t-\tau)} d \tau$ is approximated by the trapezoidal rule. More precisely, if $\left\{t_{i}=i h, i=\overline{1, M+1}\right\}$ is a subdivision of $[0, T]$ and $f^{h}(t)=\sum_{i=1}^{M+1} f_{i} \varphi\left(t-t_{i}\right)$ is an interpolation of $f$, where

$$
\varphi(t)= \begin{cases}1+\frac{t}{h}, & -1 \leq t \leq 0 \\ 1-\frac{t}{h}, & 0<t \leq 1\end{cases}
$$

is a basic function, and $f_{i}=f\left(t_{i}\right)$, then $g_{n}$ is approximated by

$$
g_{n}^{h}(t)=\sum_{i=1}^{M+1} g_{n, i} \varphi\left(t-t_{i}\right), \quad g_{n, i}=\sum_{j<i} c_{i j}\left(s_{n}\right) f_{j}
$$

with $c_{i j}(s)=\frac{2}{h s^{2}} e^{(i-j) h s}[\cosh (s h)-1]$. 


\subsection{Approximation by finite difference method (FDM)}

Problem (5) can be discretized by replacing the derivatives by difference quotients as follows. Consider a uniform grid points in the $(r, t)$ plane:

$$
\begin{cases}r_{i}=a+(i-1) h, & i=\overline{0, N+1}, h=(b-a) / N \\ t_{n}=n \tau, & n=\overline{0, M+1}, \tau=T / M .\end{cases}
$$

Letting $u_{i}^{n}=u\left(r_{i}, t_{n}\right)$, system (5) is discretized by the following finite difference scheme:

$$
\begin{cases}\frac{u_{i}^{n+1}-u_{i}^{n}}{\tau}=\frac{1}{h^{2}}\left(u_{i+1}^{n}-2 u_{i}^{n}+u_{i-1}^{n}\right)+\frac{1}{2 r_{i} h}\left(u_{i+1}^{n}-u_{i-1}^{n}\right), & i=1, \ldots, N, n=1, \ldots, M, \\ u_{i}^{0}=0, & i=1, \ldots, N, \\ u_{0}^{n}=f_{n}, u_{N+1}^{n}=u_{N}^{n}, & n=1, \ldots, M .\end{cases}
$$

Scheme (23) is explicit, and the solution $u_{i}^{n+1}$ is easily found:

$$
u_{i}^{n+1}=R\left(1+\frac{h}{2 r_{i}}\right) u_{i+1}^{n}+(1-2 R) u_{i}^{n}+R\left(1-\frac{h}{2 r_{i}}\right) u_{i-1}^{n} \quad \text { with } R=\frac{\tau}{h^{2}} .
$$

We can prove the following result concerning the stability of scheme (23).

\section{Theorem 3}

(1) The finite difference scheme (23) is consistent of order $O\left(\tau+h^{2}\right)$.

(2) If

$$
R=\frac{\tau}{h^{2}} \leq \frac{1}{2} \quad(\text { with } h<1)
$$

then scheme (23) is $L^{\infty}$-stable.

Proof (1) Assuming that the solution is fairly regular $\left(C^{4}\right)$, we use the Taylor expansion.

(2) This can be proved in a similar way as in the lecture [16]. If $R=\frac{\tau}{h^{2}} \leq \frac{1}{2}$, then we see from (24) that $u_{i}^{n+1}$ is a convex combination of $u_{i-1}^{n}, u_{i}^{n}, u_{i+1}^{n}$. Letting $M^{n}=\max _{i=1, \ldots, N}\left(u_{i}^{n}\right)$, we have

$$
u_{i}^{n+1} \leq R\left(1-\frac{h}{2 r_{i}}\right) M^{n}+(1-2 R) M^{n}+R\left(1+\frac{h}{2 r_{i}}\right) M^{n}
$$

from which it follows that $u_{i}^{n+1} \leq M^{n}$. Taking the maximum, we deduce that $M^{n+1} \leq M^{n}$. In the same way, we set $m^{n}=\min _{i=1, \ldots, N}\left(u_{i}^{n}\right)$. Then

$$
u_{i}^{n+1} \geq R\left(1-\frac{h}{2 r_{i}}\right) m^{n}+(1-2 R) m^{n}+R\left(1+\frac{h}{2 r_{i}}\right) m^{n}
$$

and $u_{i}^{n+1} \geq m^{n}$. Taking the minimum, we obtain $m^{n+1} \geq m^{n}$. Hence

$$
\max _{i=1, \ldots, N}\left(u_{i}^{n+1}\right) \leq \max _{i=1, \ldots, N}\left(u_{i}^{0}\right) \text { and } \min _{i=1, \ldots, N}\left(u_{i}^{n+1}\right) \geq \min _{i=1, \ldots, N}\left(u_{i}^{0}\right),
$$

which establishes the stability. 
Figure 1 Comparison of exact solution $g_{\mathrm{ex}}$ and numerical solution $g_{\text {ap }}$ of the direct problem; (a) Test 1; (b) Test 2.
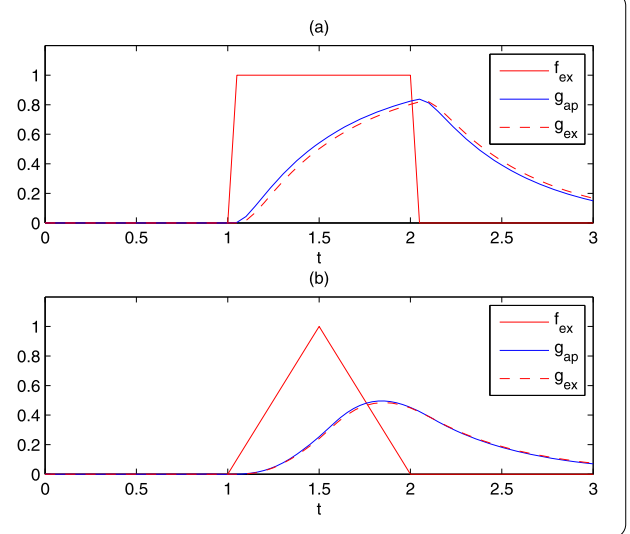

\subsection{Numerical examples}

Put $a=1, b=2, T=3$.

We consider the following examples.

Test 1 Data: $u(a, t)=f(t)=\chi_{[1,2]}\left(\chi_{I}\right.$ denotes the characteristic function of an interval $\left.I\right)$.

\section{Test 2}

$$
f(t)= \begin{cases}2(t-1), & 1<t<1.5 \\ 2(2-t), & 1.5<t<2 \\ 0 & \text { otherwise }\end{cases}
$$

In the following figures, we show the response $g(t)=u(b, t)$ to the source $f(t)$.

In Figure 1, we call $g_{\text {ex }}$ the solution given by the truncated series (17) (with a rank $N \geq$ $40)$ and $g_{\text {ap }}$ the approximate solution computed by FDM with parameters $N=30$ and $M=12,000$.

\section{Resolution of the inverse problem}

\subsection{Integral equation}

As the function $u(b, t)=g(t)$ can be known, the resolution of the inverse problem (1)-(2) can be reduced to the resolution of the Volterra integral equation of the first-kind

$$
A f(t):=\int_{0}^{t} k(t-\tau) f(\tau) d \tau=g(t)
$$

with the kernel

$$
k(t)=\sum_{n=1}^{\infty} \beta_{n} \frac{W\left(b, s_{n}\right)}{W^{\prime}\left(a, s_{n}\right)} e^{s_{n} t} .
$$

The function $k(t)$ is continuous on $\left[0,+\infty\right.$ [ satisfying $f(0)=0,|k(t)| \leq C_{1} e^{-C_{2} t}$, where $C_{1}$, $C_{2}$ two constants, for $t \geq 1$ and $k \in C^{\infty}(] 0,+\infty[)$. Then $A \in \mathcal{L}(H), H=L^{2}([0, T])$. The range $\mathcal{R}(A)$ of $A$ is nonclosed in $H$ ( $A$ is compact and nondegenerate). This means that equation (25) is ill-posed. The problem is, moreover, severely ill-posed since all derivatives 
of the kernel $k(t)$ vanish to zero according to Lemma 1(ii) (see also [17]). Therefore, some kind of regularization procedure will be necessary to solve the problem in the case of a perturbed data $g^{\delta}$.

For the numerical resolution of equation (25), we approximate the kernel $k$ by the truncated series

$$
k_{N}(t)=\sum_{n=1}^{N} \beta_{n} \frac{W\left(b, s_{n}\right)}{W^{\prime}\left(a, s_{n}\right)} e^{s_{n} t}
$$

Remark 2 The truncation error (the rest $R_{N}=k-k_{N}$ of the series) is estimated by

$$
\begin{aligned}
\left|R_{N}(t)\right| & \leq C_{1} \sum_{n \geq N} n e^{-C_{2} n^{2} t} \\
& \leq C_{1} \sum_{n \geq N} n e^{-C_{2} n \epsilon} \\
& \leq \frac{C_{1} N e^{-C_{2} \epsilon N}}{\left(1-e^{-C_{2} \epsilon}\right)^{2}} \quad \text { for } N t \geq \epsilon
\end{aligned}
$$

with $C_{2}=\left(\frac{\pi}{\lambda-1}\right)^{2}$. This means that, for $t$ close to 0 , we need more terms in the series. For computation, we choose $N=40$ for $h<t \leq 0.1, N=20$ for $0.1 \leq t \leq 1$, and $N=10$ for $t \geq 1$. For $0 \leq t \leq h$, we can take $k(t)=0$, since we know from (13) that $k(b, t)$ is close to zero as $t \rightarrow 0$ (see Figure 2). The parameter $h=\frac{T}{M}$ is the step time (we assume that $h \geq \frac{\epsilon}{N}$ ).

We denote by $A_{N}$ the operator with kernel $k_{N}$.

Proposition $1 A_{N}$ converge to $A$ in the Banach space $\mathcal{L}(X), X=C[0, T]$ equipped with the norm $\|\cdot\|_{\infty}$.

Proof Using the asymptotic formula (19) with $r=b$, we can write

$$
R_{N}(t)=\sum_{n \geq N}\left((-1)^{n} \frac{\beta_{n}}{\sqrt{\lambda}(1-\lambda)}+\gamma_{n}\right) e^{-\beta_{n}^{2} t}
$$

Figure 2 The heat kernel $k_{N}(b, t)$ with $N=40$.

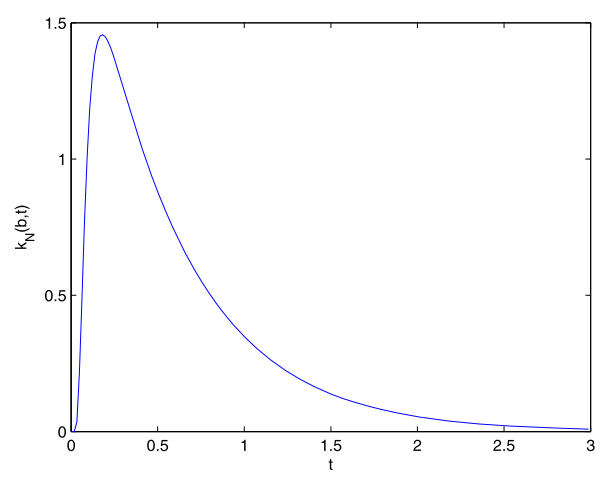


with $\gamma_{n}$ bounded. Then

$$
\left|R_{N}(t)\right| \leq C_{3} \beta_{N} e^{-\beta_{N}^{2} t}+C_{4} \sum_{n \geq N} e^{-\beta_{n}^{2} t}, \quad C_{4}=\sup \left|\gamma_{n}\right| .
$$

Using (27) with $\epsilon=1$, it follows that

$$
\begin{aligned}
\int_{0}^{T}\left|R_{N}(t)\right| d t & =\int_{0}^{1 / N}\left|R_{N}(t)\right| d t+\int_{1 / N}^{T}\left|R_{N}(t)\right| d t \\
& \leq C_{3} \int_{0}^{1 / N} \beta_{N} e^{-\beta_{N}^{2} t} d t+O\left(N e^{-C_{2} N}\right)=O\left(1 / \beta_{N}\right)
\end{aligned}
$$

which leads to

$$
\forall t \in[0, T], \quad\left|\left(A-A_{N}\right) f(t)\right| \leq\|f\|_{\infty} \int_{0}^{T}\left|R_{N}(t)\right| d t,
$$

and $\left\|A-A_{N}\right\|_{\mathcal{L}(X)}=O(1 / N)$.

\subsection{Tikhonov regularization}

The approximate equation $A_{N} f=g$ is solved by the Tikhonov regularization method. Recall the principle of the method.

Suppose that $A \in \mathcal{L}(H)$ is a compact operator in Hilbert space, injective and with dense range. The equation $A f=g$ is ill posed, that is, $A^{-1}: \mathcal{R}(A) \rightarrow H$ is not bounded (a small error in data $g$ generates an important perturbation on the computed solution $f$ ). The Tikhonov regularization method consists in solving the normal equation

$$
\left(A^{\star} A+\alpha I\right) f^{\alpha}=A^{\star} g,
$$

where $\alpha>0$ is a regularization parameter, and $I$ is the identity operator. Equivalently, $f^{\alpha}$ is the unique minimum of the Tikhonov functional

$$
J^{\alpha}[f]=\|A f-g\|_{H}^{2}+\alpha\|f\|_{H}^{2} .
$$

The solution $f^{\alpha}$ can be written as follows:

$$
f^{\alpha}=\left(A^{\star} A+\alpha I\right)^{-1} A^{\star} g,
$$

which can be expressed in terms of the singular system $\left(\mu_{j}, u_{j}, v_{j}\right)_{j \in \mathrm{N}^{*}}$ as

$$
f^{\alpha}:=R_{\alpha} g=\sum_{j} \frac{\mu_{j}}{\mu_{j}^{2}+\alpha}\left(g, v_{j}\right) u_{j}
$$

Now let $g \in \mathcal{R}(A)$, and let $g^{\delta} \in H$ be a measured data with $\left\|g-g^{\delta}\right\|_{H} \leq \delta$. We define $f^{\alpha, \delta}=R_{\alpha} g^{\delta}$. A posteriori discrepancy principle gives a selection of $\alpha$ (a solution of the equation $\left\|A f^{\alpha, \delta}-g^{\delta}\right\|_{H}=\delta$ ). Standard Tikhonov regularization theory (see [18, Theorem 2.17]) shows the convergence $f^{\alpha, \delta} \rightarrow f$ in $H$ as $\delta \rightarrow 0$ with the strategy $\alpha=\alpha(\delta)$. For other methods, we indicate the papers $[17,19]$, which give a survey of regularization methods for first-kind Volterra equations. 


\subsection{Numerical experiment}

We consider the following examples.

Test 1 As a first example, we consider the couple $\{f, g\}$, where

$$
u(a, t)=f(t)=\chi_{[1,2]}
$$

and $g(t)=u(b, t)$ is computed by finite difference scheme (see Section 2.2).

Test 2 We consider the example given by

$$
f(t)= \begin{cases}2(t-1), & 1<t<1.5 \\ 2(2-t), & 1.5<t<2 \\ 0 & \text { otherwise }\end{cases}
$$

To check the efficiency of the proposed algorithm, we choose in numerical experiments the parameters $a=1, b=2, T=3$ or $T=5$ as required, and the rank of truncation $30 \leq$ $N \leq 40$.

For an exact data function $g(t)=u(b, t)$, we use a finite difference scheme with $N=30$ points in the interval $[1,2]$ and $M=12,000$ points in $[0, T]$. The discrete noisy version is $g^{\delta}=g+\delta \operatorname{randn}(\operatorname{size}(g))$, the command 'randn $(\cdot)$ ' generates arrays of random numbers whose elements are normally distributed with mean 0 , variance $\sigma^{2}=1$, and standard deviation $\sigma=1$. For the singular decomposition and Tikhonov-Morozov algorithms, we used the Matlab package developed by Hansen [20].

\subsection{Results and discussion}

Figures 3 and 4 show the numerical results that confirm the stability of the method with respect to the noise level $\delta \leq 0.001$. However, the rank in $k_{N}$ must be large enough (here $N \geq 30$ ) to ensure the convergence of the Tikhonov algorithm. Figure 5(a) shows that, for $\delta=0.01$, the oscillations increase, which requires a new regularization. Indeed, if we use the mollification method [4], then the oscillations are damped (see Figure 5(b)). The operation consists in taking the convolution $g_{v}=\rho_{v} * g$ with $\rho_{v}(t)=\frac{1}{v \sqrt{\pi}} \exp \left(-\frac{t^{2}}{v^{2}}\right)$, where $v \rightarrow 0$ is the radius of mollification. If $g$ vanishes near the ends of the interval $[0, T]$, then $g_{v}$ is a smooth function and is a good approximation of $g$; this fact is realized if the observation time $T$ is large enough (in practice, we take $T=2 T_{0}$ if supp $f \subset\left[0, T_{0}\right]$ ). In the presence of the noise, according to the analysis in [4], we choose $v=c \sqrt{\delta}$ with $c=1$ estimated by test.

\section{Conclusion}

In this paper, we considered the inverse boundary-value problem of heat conduction with radial variable (in the cylindrical domain). The problem is solved by the approach based on the direct and inverse Laplace transforms. This leads to the Volterra equation of the first-kind with a special kernel. The Tikhonov method is applied to solve numerically this equation for perturbed data. The numerical results show that the method is efficient when the noise level $\delta=0.001$. If $\delta=0.01$, then the mollification procedure is applied (before the regularization) to the data $g^{\delta}$, but with time observation $T$ large enough. 
Figure 3 Test 1. Exact and approximate solution of the inverse problem (IP); (a) with $(\delta=0, N=40$ and $T=3)$; (b) with $(\delta=0.001, N=40, T=3)$.
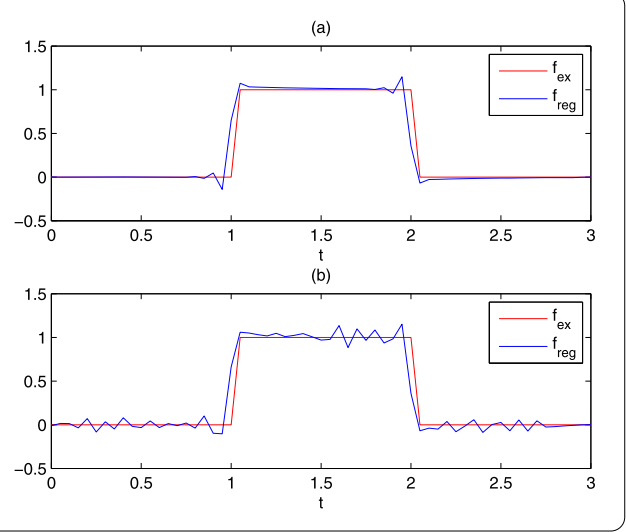

Figure 4 Test 2. Exact and approximate solution of IP; (a) with $(\delta=0, N=40, T=3)$; (b) with $(\delta=0.001$, $N=50, T=3)$.
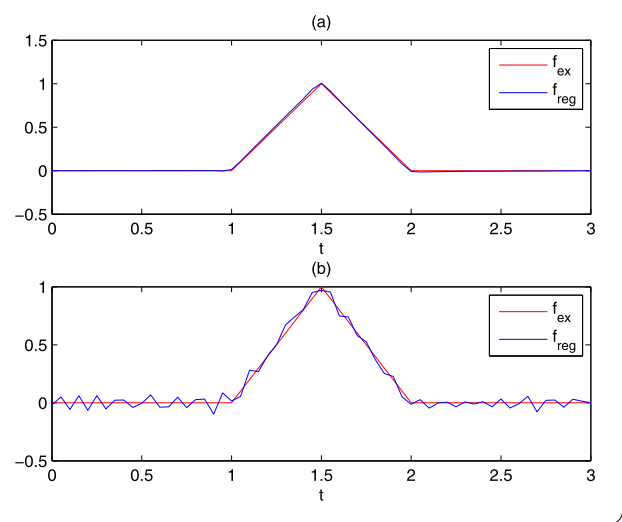

Figure 5 Test 2. Exact and approximate solution of IP with parameters $(\delta=0.01, N=30, T=5)$;

(a) without mollification; (b) with mollification $v=0.1$.
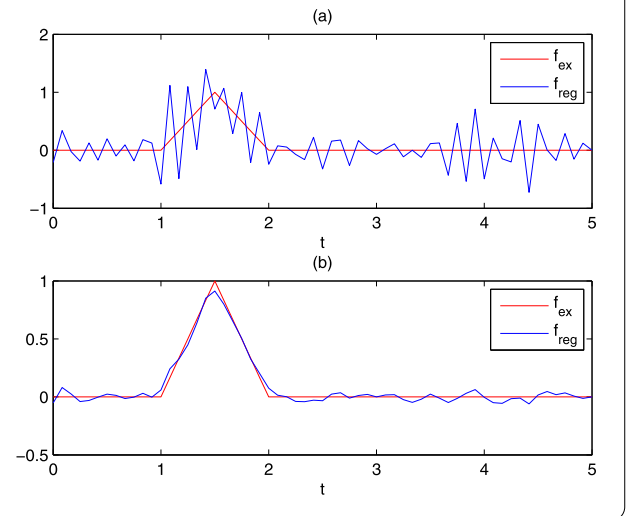

\section{Appendix 1: Asymptotic expansions}

For fixed $v$ and $|z| \rightarrow \infty$, we have [15]

$$
\begin{aligned}
& J_{v}(z) \sim \sqrt{\frac{2}{\pi z}} \cos \left(z-\frac{\pi}{4}-\frac{v \pi}{2}\right), \quad Y_{\nu}(z) \sim \sqrt{\frac{2}{\pi z}} \sin \left(z-\frac{\pi}{4}-\frac{\nu \pi}{2}\right), \\
& I_{v}(z) \sim \frac{e^{z}}{\sqrt{2 \pi z}}, \quad K_{v}(z) \sim \sqrt{\frac{\pi}{2 z}} e^{-z} .
\end{aligned}
$$


We consider the rapport $G(r, s)=\frac{W(r, s)}{W(a, s)}$ with $W(r, s)=I_{1}(b \sqrt{s}) K_{0}(r \sqrt{s})+K_{1}(b \sqrt{s}) I_{0}(r \sqrt{s})$. Due to (30), we have, for $r \in] a, b]$ and $|s| \rightarrow+\infty$,

$$
|G(r, s)|=\sqrt{\frac{a}{r}} \exp \left(-(r-a) \rho^{1 / 2}\left|\cos \frac{\theta}{2}\right|\right)+O\left(\frac{1}{\rho}\right)
$$

where $\rho=|s|$ and $\theta=\arg (s) \in\left[-\frac{\pi}{2}, \frac{3 \pi}{2}\right]$. Then there exist positive constants $C$ and $\mu$ such that, for $|s|$ large enough, say $|s| \geq \rho_{0}$,

$$
\begin{aligned}
\left|G(r, s) e^{s t}\right| & \leq C \exp \left[-(r-a) \rho^{1 / 2}\left|\cos \frac{\theta}{2}\right|+t \rho \cos \theta\right] \\
& \leq C \exp \left[-m \sqrt{\rho}\left(\left|\cos \frac{\theta}{2}\right|+|\cos \theta|\right)\right] \quad(m=\min (r-a, t)) \\
& \leq C \exp (-\mu \sqrt{\rho}) \quad\left(\text { uniformly for } \theta \in\left[\frac{\pi}{2}, \frac{3 \pi}{2}\right]\right) .
\end{aligned}
$$

\section{Appendix 2: Zeros of cross-product}

We want to seek the roots $s_{n}$ of the following function (cross-product):

$$
W(s)=I_{1}(b \sqrt{s}) K_{0}(a \sqrt{s})+K_{1}(b \sqrt{s}) I_{0}(a \sqrt{s}) .
$$

We show that $s_{n}=-\beta_{n}^{2}$ with $\beta_{n} \rightarrow+\infty$ as $n \rightarrow+\infty$. For this, we consider the self-adjoint operator defined in $H=L^{2}((a, b) ; r d r)$ by

$$
A U=-\frac{1}{r} \frac{d}{d r}\left(r \frac{d U}{d r}\right), \quad D(A)=\left\{U \in H^{2}(] a, b[) ; U(0)=U^{\prime}(1)=0\right\} .
$$

If $A U=\lambda U$, then $(A U, U)=\left\|U^{\prime}\right\|^{2}=\lambda\|U\|^{2}$. Since $A^{-1}$ is compact, $A$ has a sequence of positive eigenvalues $\lambda_{n} \rightarrow+\infty$. On the other hand $\lambda_{n}=-s_{n}$ coincides with the roots of $W(s)$. Indeed, this can be seen by solving the Sturm-Liouville problem (system (6) in Section 2) with $\lambda=-s$ and $F=0$.

Now we give the behavior of $s_{n}$. Using the relations between the Bessel functions

$$
I_{n}(z)=i^{-n} J_{n}(i z) \quad \text { and } \quad K_{n}(z)=\frac{\pi}{2} i^{n+1}\left[J_{n}(i z)+i Y_{n}(i z)\right] \quad \text { for } n \in \mathrm{N}
$$

we obtain, for $s=-\beta^{2}$,

$$
W\left(-\beta^{2}\right)=\frac{i \pi}{2}\left[J_{0}(a \beta) Y_{1}(b \beta)-J_{1}(b \beta) Y_{0}(a \beta)\right] .
$$

Using the asymptotic expansions (29) for large $\beta$, we get

$$
W\left(-\beta^{2}\right) \sim \frac{i}{\sqrt{a b} \beta} \cos \left[\left(\frac{b}{a}-1\right) \beta\right]
$$

and hence the zeros of $W(s)$ are a sequence $s_{n}=-\beta_{n}^{2}, n=1,2, \ldots$, such that

$$
\beta_{n} \sim\left(n-\frac{1}{2}\right) \frac{a \pi}{b-a} \quad \text { as } n \rightarrow+\infty
$$


Acknowledgements

The work is supported by the National Research Foundation (CNEPRU) of Algeria (No. B01120120016).

\section{Competing interests}

The authors declare that they have no competing interests.

\section{Authors' contributions}

All authors contributed equally to the writing of this paper. All authors read and approved the final manuscript.

\section{Publisher's Note}

Springer Nature remains neutral with regard to jurisdictional claims in published maps and institutional affiliations.

Received: 12 July 2017 Accepted: 21 October 2017 Published online: 02 November 2017

\section{References}

1. Eldén, L, Berntsson, F, Regińska, T: Wavelet and Fourier methods for solving the sideways heat equation. SIAM J. Sci. Comput. 21(6), 2187-2205 (2000)

2. Berntsson, F: A spectral method for solving the sideways heat equation. Inverse Probl. 15, 891-906 (1999)

3. Fu, C-L: Simplified Tikhonov and Fourier regularization methods on a general sideways parabolic equation. J. Comput. Appl. Math. 167(2), 449-463 (2004)

4. Murio, DA: The Mollification Method and the Numerical Solution of IIl-Posed Problems. Wiley, New York (1993)

5. Yaparova, N: Numerical methods for solving a boundary-value inverse heat conduction problem. Inverse Probl. Sci. Eng. 22(5), 832-847 (2014)

6. Cheng, W, Fu, C-L: Solving the axisymmetric inverse heat conduction problem by a wavelet dual least-squares method. Boundary Value Problems 2009, Article ID 260941 (2009). doi:10.1155/2009/260941

7. Cheng, W, Fu, C-L: Two regularization methods for an axisymmetric inverse heat conduction problem. J. Inverse III-Posed Probl. 17(2), 159-172 (2009)

8. Cheng, W: Regularization and stability estimates for an inverse source problem of the radially symmetric parabolic equation. J. Inequal. Appl. 2015, 136 (2015)

9. Cheng, W, Zhao, L-L, Fu, C-L: Source term identification for an axisymmetric inverse heat conduction problem. Comput. Math. Appl. 59, 142-148 (2010)

10. Xiong, X-T: On a radially symmetric inverse heat conduction problem. Appl. Math. Model. 34, 520-529 (2010)

11. Cheng, W, Fu, C-L: A modified Tikhonov regularization method for an axisymmetric backward heat equation. Acta Math. Sin. Engl. Ser. 26(11), 2157-2164 (2010)

12. Johansson, BT, Lesnic, D, Reeve, T: A method of fundamental solutions for the radially symmetric inverse heat conduction problem. Int. Commun. Heat Mass Transf. 39, 887-895 (2012)

13. Tuan, NH, Kirane, M, Luu, VCH, Bin-Mohsin, B: A regularization method for time-fractional linear inverse diffusion problems. Electron. J. Differ. Equ. 2016, 290 (2016)

14. Ditkine, V, Proudnikov, A: Transformation Intégrales et Calcul Opérationnel. Traduit du Russe. Éditions MIR, Moscou (1978)

15. Abramowitz, M, Stegun, IA: Handbook of Mathematical Functions. Dover, New York (1972)

16. Herbin, R: Analyse numérique des équations aux dérivées partielles. Engineering school, Marseille 2011. https://cel.archives-ouvertes.fr/cel-00637008

17. Lamm, PK: A survey of regularization methods for first-kind Volterra equations. In: Colton, D, Engl, HW, Louis, AK, McLaughlin, JR, Rundell, W (eds.) Surveys on Solution Methods for Inverse Problems, pp. 53-82. Springer, Vienna (2000). doi:10.1007/978-3-7091-6296-5_4

18. Kirsh, A: An Introduction to the Mathematical Theory of Inverse Problems. Applied Mathematical Sciences Book Series, vol. 120. Springer, Berlin (2011)

19. Lamm, PK, Eldén, L: Numerical solution of first-kind Volterra equations by sequential Tikhonov regularization. SIAM J. Numer. Anal. 34(4), 1432-1450 (1997)

20. Hansen, PC: Regularization tools version 4.0 for Matlab 7.3. Numerical Algorithms 46, 189-194 (2007). doi:10.1007/s11075-007-9136-9

\section{Submit your manuscript to a SpringerOpen ${ }^{\circ}$ journal and benefit from:}

- Convenient online submission

- Rigorous peer review

- Open access: articles freely available online

- High visibility within the field

Retaining the copyright to your article

Submit your next manuscript at $>$ springeropen.com 